IASSNS-HEP-93/75

\title{
Recent developments in chiral gauge theories: Approach of infinitely many fermi fields
}

\author{
Rajamani Narayanan ${ }^{\mathrm{a} * \dagger}$

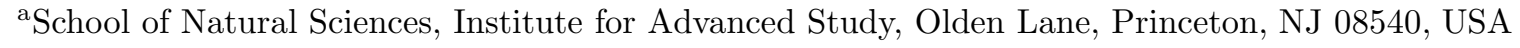

I present the recent developments in a specific sub-field of chiral gauge theories on the lattice. This subfield pertains to the use of infinitely many fermi fields to describe a single chiral field. In this approach, both anomalous and anomaly free theories can be discussed in equal footing. It produces the correct anomaly in the continuum limit. It has the potential to describe fermion number violating processes in the presence of a gauge field background with non-trivial topological charge on a finite lattice.

\section{INTRODUCTION}

Establishing the existence of chiral gauge theories outside perturbation theory, with fermionic matter in an anomaly-free complex representation of the gauge group, is a long standing open problem in lattice field theory. Many approaches have been proposed and analyzed in the past and this topic has been an active field of research as is evident from the review talks in the past lattice conferences [1]. Unfortunately, the success has been very limited. The difficulty, as everyone present in the conference knows very well, is a conceptual one and is not the absence of fast enough computers [2].

The conceptual difficulty arises from the presence of anomalies in a chiral gauge theory. This makes the regularization a very subtle problem. This is a well understood issue in the realm of perturbation theory. There has also been considerable progress in the past to elevate the continuum regularization to a non-perturbative level. This has been very useful in bringing out the subtleties present in chiral gauge theories. But a lattice regularization of chiral gauge theories is needed to provide a mundane framework for definite computations. It is needed to understand the contin-

*Plenary talk presented at the Lattice '93 conference held between October 12-16 in Dallas, Texas

${ }^{\dagger}$ This work was supported in part by the DOE grant \#DEFG02-90ER40542. uum limit of chiral gauge theories, particularly the difference between anomalous and anomaly free theories. It is needed to non-perturbatively compute 't Hooft processes [3].

I shall deviate from the conventional line of thought right from the start. The difficulties encountered by the previous approaches will greatly influence the nature of questions that I address here. The hope is that this review will complement previous reviews that dealt with chiral fermions and facilitate future progress in the understanding of chiral theories. The line of thought is strongly influenced by two papers: one by Kaplan [4] which many of you are probably familiar with; and the second, a lesser known paper by Frolov and Slavnov [5]. Several papers have been written on the pros and cons of Kaplan's proposal in the last one year 6 12]. I shall try my best to combine most of the ideas in these papers to present a coherent view of the new proposal to tackle chiral gauge theories.

The organization of the talk is as follows. I will start by showing how one can use an infinite set of fermi fields to describe a single chiral fermion in the continuum 5 . I I will then show that this can be extended to the lattice. The resulting model will be different from the one proposed by Kaplan [4] in that the gauge fields will be four dimensional and not five dimensional. I will briefly discuss the difficulties in working with five dimensional gauge fields. The need for infinitely many 
fermi fields seems to make the model impractical at first sight. I will show that some attempts to use a finite set of fields destroys the chiral nature of the theory and makes it vector-like [12]. Next, I will show how one can deal with an infinite number of fermi fields in a practical calculation without restricting them to a finite set. I will focus on the effective action induced by fermions and will show that it is an overlap of two second quantized ground states. This provides a non-perturbative formula for the effective action on a finite lattice whose merits can be explicitly tested. At this point, I will make a formal extension of the overlap to the continuum and show how this formula is a definition of the "chiral determinant" in the continuum. Since the effective action is an overlap of two different states, there is an ambiguity in its imaginary part. I will show that perturbation theory provides a way of fixing this ambiguity. Finally, I will show that because the effective action is an overlap of two ground states, it has the potential to vanish exactly for some gauge backgrounds. Further, insertion of an appropriate number of fermion operators in between the two ground states will make it non-zero in such gauge backgrounds. In this way, I will obtain the 't Hooft picture [3] on the lattice and also get a definition for the topological charge that is always an integer.

\section{INFINITELY MANY FERMI FIELDS}

Consider the Pauli Villars regularization of a single chiral fermion transforming under a complex representation of the gauge group G. A general, local, hermitian Lagrangian for the fermions is

$$
\begin{array}{ll}
\mathcal{L}= & i \bar{\psi}_{s} \gamma^{\mu}\left(\partial_{\mu}-i g A_{\mu}\right) \psi_{s} \\
+ & \bar{\psi}_{s}\left(M_{s s^{\prime}} P_{+}+M_{s s^{\prime}}^{\dagger} P_{-}\right) \psi_{s^{\prime}}
\end{array}
$$

$\psi_{s}$ is a set of $s$ Dirac fields which includes the original chiral fermion and as many regulator fields as is needed. $P_{ \pm}=\frac{1}{2}\left(1 \pm \gamma^{5}\right)$ are the projectors into right and left handed fields respectively. $A_{\mu}=A_{\mu}^{a} T^{a}$ with $T^{a}$ being the group generators in some complex representation. $M$ is some mass matrix. The structure, $\gamma_{0}\left(M P_{+}+M^{\dagger} P_{-}\right)$is hermitian. To describe a single right handed chiral

fermion, $M$ should have one zero mode and $M^{\dagger}$ should not have any, i.e, $M$ should have an index equal to unity. This cannot be achieved by a finite dimensional matrix $M$. Restriction to a finite set of fields will force the chiral fermions to occur in pairs of opposite chirality. If $M$ is infinite dimensional, one can construct an $M$ with non-zero index. A simple example is to choose $M$ proportional to the annihilation operator of an harmonic oscillator where $s$ plays the role of the label associated with the energy levels of the harmonic oscillator. All the heavy modes of $M$ and all the modes of $M^{\dagger}$ correspond to regulator fields and should be taken to infinity. One can now proceed to see if the statistics of the infinitely many fields can be suitably chosen to obtain a well regulated theory. It can be shown that we will end up with a well regulated theory (in the perturbative sense) if the original theory is anomaly free 河, 6 .

To extend the above idea to a non-perturbative level, consider the lattice regularization of the same problem. One can start with a Langrangian similar to (1) and extend it to the lattice in the usual manner. Now, $M$ should satisfy the following properties. In the region enclosing $p=0$ in the Brillouin zone, $M$ should have an index equal to unity. But the zero mode of $M$ should disappear as one goes near the edges of the Brillouin zone where the potential doublers exist. One can achieve such an $M$ by considering an infinite set of Wilson fermions with a $p$ independent mass matrix coupling them. A specific action for fermions that achieves this goal is

$$
\begin{aligned}
S_{F}(\bar{\psi}, \psi, U)= & \frac{1}{2} \sum_{n, s, \mu} \bar{\psi}_{n, s}\left(1+\gamma^{\mu}\right) U_{n, \mu} \psi_{n+\hat{\mu}, s} \\
& +\frac{1}{2} \sum_{n, s, \mu} \bar{\psi}_{n, s}\left(1-\gamma^{\mu}\right) U_{n-\hat{\mu}, \mu}^{\dagger} \psi_{n-\hat{\mu}, s} \\
& +\frac{1}{2} \sum_{n, s} \bar{\psi}_{n, s}\left(1+\gamma^{5}\right) \psi_{n, s+1} \\
& +\frac{1}{2} \sum_{n, s} \bar{\psi}_{n, s}\left(1-\gamma^{5}\right) \psi_{n, s-1} \\
& -\sum_{n, s}\left[5-m \operatorname{sign}\left(s+\frac{1}{2}\right)\right] \bar{\psi}_{n, s} \psi_{n, s}
\end{aligned}
$$


$\bar{\psi}_{n, s}$ and $\psi_{n, s}$ are Dirac spinors. $m$ is a parameter in the region $0<m<1$. $n$ is a four component integer labeling sites on the lattice and $s$ labels the infinite copies of fermions. $n$ can run over a finite set or an infinite set of integers. When it runs over a finite set, which will be the case of practical interest, the boundary conditions on the fermions will be chosen to be anti-periodic. The above action will be referred to as the "wall" realization of a single chiral fermion. Although, this is basically the idea of Kaplan [4], the line of thought presented here is significantly different from his and the action itself has some differences.

Kaplan [4] viewed the infinite set of fermi fields as residing in a fifth direction. As such, he was originally inclined to couple then to a five dimensional gauge field in the spirit of Callan and Harvey [13]. This introduces a lot of unwanted degrees of freedom. Wild fluctuations of the gauge field in the fifth direction could essentially decouple adjacent $s$ slices and spoil the chiral zero mode. It is shown in the context of mean field analysis that increasing the coupling in the fifth direction brings about a layered phase [10,12]. Perturbative analysis of a $2+1$ (two physical and one $s$ direction) model with three dimensional $\mathrm{U}(1)$ gauge fields has been done 11. Here one obtains the parity-odd terms that break 2-D gauge invariance but one also gets a longitudinal piece in the real part that does not vanish even for anomaly free cases. These investigations seem to indicate that working with higher dimensional gauge fields is not a good direction to proceed. In (2) all the fermi fields are coupled to the same gauge field. This is in the same spirit as in PauliVillars regularization. The gauge fields are therefore the usual four dimensional fields.

Kaplan, in a later proposal [14], did suggest a model with four dimensional gauge fields. But he made the fifth direction finite because having an infinite direction seems to make a numerical simulation impossible at first sight. Making the fifth direction finite results in the theory having two chiral modes of opposite chirality. This is called the "wall-antiwall" model, where one zero mode is centered on the wall and the other zero mode is centered on the anti-wall. To decouple one of the zero modes from the gauge field, Kaplan decided to put in gauge fields only on the slices around the wall and not have gauge fields near the antiwall. The hope was that if the wall and anti-wall were far removed from each other the resulting theory in the vicinity of the wall would be a chiral gauge theory. Gauge invariance is broken in this model and was restored by the addition of scalar fields on the two $s$ slices where the gauge field is cut-off. This model was analyzed in some detail by Golterman et.al. [12]. The introduction of the scalar fields to restore gauge invariance introduces Yukawa coupling. For zero Yukawa coupling they found two more massless modes centered on the slices where the scalar field is introduced. A search for a region of the parameter space where the scalar field and the extra zero modes are all decoupled from the physical zero mode was not met with success. There is no obvious reason why this model should not succeed nor was the search performed in [12] exhaustive enough to rule out this approach. In the next section, I will show that it is possible to deal with an infinite set of fields directly and still be able to deal with the problem numerically in a computer with finite memory size in a finite amount of time!

There are two other technical differences between (2) and Kaplan's proposal. One is that the Wilson coefficient is set to $r=0.5$. This is to ensure positivity in the transfer matrix formalism. The other difference is that the defect is between $s=0$ and $s=-1$. This simplifies the result obtained from the transfer matrix formalism.

\section{THE OVERLAP FORMULA}

In this section, I will discuss the implementation of the "wall" realization on a finite lattice by keeping the number of fermi fields infinite. I will concentrate only on the effective action induced by the fermions and present the arguments and the results. For derivations, the reader is referred to [7].

Consider the action in (2) on a $L^{4} \times \infty$ lattice. The lattice is finite in the four spatial directions and is infinite in the $s$ "direction". In each slice $s$ there are a finite number of degrees of freedom, both fermionic and bosonic. Further, the gauge 
fields are the same in all slices. The transfer matrix is obtained by the standard translation of the Grassmann integration into the operator formalism. We will choose the propagation along the $s$ direction and the transfer matrix will connect two adjacent $s$ slices. Since the action in (2) is uniform for $s<0$ and for $s \geq 1$, the transfer matrices connecting slices $s$ for $s<0$ will all be the same and so will the transfer matrices connecting slices $s$ for $s \geq 1$. This implies that there are only two finite dimensional transfer matrices in the problem. The result for the effective action, $S_{\text {eff }}(U)$, is

$$
\begin{aligned}
& e^{S_{\text {eff }}(U)}=\lim _{s \rightarrow \infty} \quad\left[\operatorname{det} \mathbf{B}^{-}\right]^{s+\frac{3}{2}}\left[\operatorname{det} \mathbf{B}^{+}\right]^{s+\frac{3}{2}} \\
& <b-\left|e^{-s \hat{H}_{-}} e^{-s \hat{H}_{+}}\right| b+> \\
& \hat{H}_{ \pm}=-\hat{a}^{\dagger} \mathbf{H}_{ \pm} \hat{a} \\
& e^{\mathbf{H}_{ \pm}}=\left(\begin{array}{cc}
\frac{1}{\mathbf{B}^{ \pm}} & \frac{1}{\mathbf{B}^{ \pm}} \mathbf{C} \\
\mathbf{C}^{\dagger} \frac{1}{\mathbf{B}^{ \pm}} & \mathbf{C}^{\dagger} \frac{1}{\mathbf{B}^{ \pm}} \mathbf{C}+\mathbf{B}^{ \pm}
\end{array}\right) \\
& \mathbf{B}_{n \alpha i, m \beta j}^{ \pm}=(5 \mp m) \delta_{n m} \delta_{\alpha \beta} \delta_{i j} \\
& -\frac{1}{2} \delta_{\alpha \beta} \sum_{\mu}\left[\delta_{m, n+\hat{\mu}} U_{n, \mu}^{i j}+\delta_{n, m+\hat{\mu}} U_{m, \mu}^{j i}{ }^{*}\right] \\
& \mathbf{C}_{n \alpha i, m \beta j}=\frac{1}{2} \sum_{\mu}\left[\delta_{m, n+\hat{\mu}} U_{n, \mu}^{i j}-\delta_{n, m+\hat{\mu}} U_{m, \mu}^{j i}{ }^{*}\right] \sigma_{\mu}^{\alpha \beta}
\end{aligned}
$$

$\hat{a}^{\dagger}$ and $\hat{a}$ are fermion operators satisfying canonical anti-commutation relations.

$$
\begin{aligned}
& \left\{\hat{a}_{n A i}, \hat{a}_{m B j}^{\dagger}\right\}=\delta_{n m} \delta_{A B} \delta_{i j} ; \\
& \left\{\hat{a}_{n A i}, \hat{a}_{m B j}\right\}=\left\{\hat{a}_{n A i}^{\dagger}, \hat{a}_{m B j}^{\dagger}\right\}=0 .
\end{aligned}
$$

The indices $A$ and $B$ run over the four spinor components whereas the indices $\alpha$ and $\beta$ run over two spinor components. The $\gamma$ matrices are $\gamma^{\mu}=$ $\left(\begin{array}{cc}0 & \sigma_{\mu} \\ \sigma_{\mu}^{\dagger} & 0\end{array}\right) ; \gamma^{5}=\left(\begin{array}{cc}1 & 0 \\ 0 & 1\end{array}\right)$, where $\sigma_{0}=i$ and $\sigma_{k} ;$ $k=1,2,3$ are the usual Pauli matrices. $\mathbf{B}^{ \pm}$are positive definite for all values of the gauge fields. $\mathbf{H}_{ \pm}$are both hermitian and traceless. $\mid b \pm>$ are the boundary conditions at $s= \pm \infty$.

As it stands, the effective action in (3) is not finite. This is due to two contributions. One comes from the two determinants $\operatorname{det} \mathbf{B}^{ \pm}$raised to infinite power. As $s \rightarrow \infty, e^{-s \hat{H}_{ \pm}}$go into the respective projectors $e^{-\left(\lambda_{ \pm}^{0}\right) s}|0 \pm><0 \pm|$ where $\mid 0 \pm>$ and $\lambda_{ \pm}^{0}$ are the ground state eigenvectors and eigenvalues of $\hat{H}_{ \pm}$respectively. The second infinity comes from $e^{-\left(\lambda_{ \pm}^{0}\right) s}$ as $s \rightarrow \infty$. These are both bulk type infinites and to remove them consider the effective actions, $S_{\text {eff }}^{ \pm}(U)$, due to the homogeneous cases obtained by replacing the coefficient of the mass term in the action (last term in (2)) by $5 \mp m$ respectively.

$e^{S_{\text {eff }}^{ \pm}(U)}=\lim _{s \rightarrow \infty}\left[\operatorname{det} \mathbf{B}^{ \pm}\right]^{2 s+3}<b \pm\left|e^{-2 s \hat{H}_{ \pm}}\right| b \pm>(5)$

Note that $S_{\text {eff }}^{ \pm}(U)$ are both real. To cancel the bulk infinities, we define the interface effective action as

$S_{i}(U)=S_{\text {eff }}(U)-\frac{1}{2}\left[S_{\text {eff }}^{+}(U)+S_{\text {eff }}^{-}(U)\right]$

From (3) and (5), as $s \rightarrow \infty$,

$e^{S_{i}(U)}=\frac{<b-|0-><0-| 0+><0+\mid b+>}{|<b-| 0->||<b+|0+>|},(7)$

and $S_{i}(U)$ is finite. Note that the boundary states at $\pm \infty$ in (3) and (5) were appropriately chosen for the inhomogeneous and homogeneous cases. Choosing periodic boundary conditions in the $s$ direction amounts to setting $|b+>=| b->$ and summing over a complete set of states. This will make the right hand side of (7) equal to $|<0-| 0+>\left.\right|^{2}$. It is real and corresponds to an effective action coming from two chiral modes of opposite chirality and is precisely what has to be avoided. The two ends, $s \rightarrow \pm \infty$, should be kept separate. The appropriate boundary states are $|b \pm>=| 0 \pm>$. This is precisely what one would pick if one is thinking of a quantum mechanical path integral. The result is the overlap formula for the effective action:

$e^{S_{i}(U)}=<0-\mid 0+>$

The above formula is finite and non-perturbative.

\section{CONTINUUM INTERPRETATION}

I will now show that the simple formula, (8), obtained via a circuitous route starting from infinitely many fermi fields, has a simple continuum 
interpretation. The contents of this section are in no way rigorous. Consider the following Hamiltonian in the continuum:

$$
-\mathbf{H}=\left(\begin{array}{cc}
m & X^{\dagger} \\
X & -m
\end{array}\right) .
$$

$X$ is the chiral Dirac operator and $\mathbf{H}$ with $m>0$ and $m<0$ are the formal continuum limits of the Hamiltonians discussed in the previous section. The states $\mid 0 \pm>$ that enter the continuum limit of (8) are the ground states obtained by filling all the negative energy states of $\mathbf{H}$ with $m>0$ and $m<0$ respectively. Let

$$
-\mathbf{H} \psi_{\lambda}=\lambda \psi_{\lambda} ; \quad \psi_{\lambda}=\left(\begin{array}{c}
u_{\lambda} \\
v_{\lambda}
\end{array}\right)
$$

be the eigenvalue equation for $\mathbf{H}$. Then

$$
\begin{aligned}
& X^{\dagger} v_{\lambda}=(\lambda-m) u_{\lambda} ; \quad X X^{\dagger} v_{\lambda}=\left(\lambda^{2}-m^{2}\right) v_{\lambda}(11) \\
& X u_{\lambda}=(\lambda+m) v_{\lambda} ; \quad X^{\dagger} X u_{\lambda}=\left(\lambda^{2}-m^{2}\right) u_{\lambda}
\end{aligned}
$$

For $m=|m|$, the complete set of negative energy solutions are given by

$\psi_{i}^{+}=N_{i}^{+}\left(X \frac{u^{(i)}}{\sqrt{X^{\dagger} X+m^{2}}+|m|} u^{(i)}\right)$

where we used (12). For $m=-|m|$, the complete set of negative energy solutions are given by

$\psi_{i}^{-}=N_{i}^{-}\left(\begin{array}{c}X^{\dagger} \frac{1}{\sqrt{X X^{\dagger}+m^{2}}+|m|} v^{(i)} \\ v^{(i)}\end{array}\right)$

where we used (11).Note that both (13) and (14) are well defined even for the zero modes of $X X^{\dagger}$ or $X^{\dagger} X$. The superscript $i$ denotes the index of the eigenvector and $N_{i}^{ \pm}$are the normalization factors. The overlap is then given by

$$
\begin{aligned}
<0-\mid 0+> & =\operatorname{det}_{i j}\left[2 N_{i}^{-} N_{j}^{+}\right. \\
& \left.<v^{(i)}, \frac{1}{\sqrt{X X^{\dagger}+m^{2}}+|m|} X u^{(j)}>\right]
\end{aligned}
$$

This expression can be interpreted as a "determinant" of $X .\left\{u^{(i)}\right\}$ is a basis set that spans the space on which $X$ acts. The action of $X$ takes it to another space for which the set $\left\{v^{(i)}\right\}$ is a basis. The inner product on the right hand side of (15) is a matrix element of $X$. The expression (15) is (partially) regularized and its phase will be the imaginary part of the effective action.

\section{THE PHASE AMBIGUITY}

(8) still has an ambiguity arising from the phase choice of the two ground states $\mid 0 \pm>$. Different choice of phases affect the imaginary part of the effective action wherein lies the anomaly content of the theory. In particular, it is not clear why the phases cannot be chosen to make the overlap purely real. This issue can be resolved by the use of continuum perturbation theory [7]. Let

$\Psi_{p}^{+}=\psi_{p}^{+}+\psi_{q}^{+} A_{q p}+\chi_{q}^{+} B_{q p}+\psi_{q}^{+} W_{q p}+\chi_{q}^{+} X_{q p}$

$\Psi_{p}^{-} \psi_{p}^{-}+\psi_{q}^{-} C_{q p}+\chi_{q}^{-} D_{q p}+\psi_{q}^{-} Y_{q p}+\chi_{q}^{-} Z_{q p} .(16)$

$\psi_{p}^{ \pm}$are eigenvectors corresponding to negative eigenvalues and $\chi_{p}^{ \pm}$are eigenvectors corresponding to positive eigenvalues of the gauge free Hamiltonians, $\mathbf{H}_{ \pm}(0)$. $A, B, C$ and $D$ are coefficients linear in the gauge field and $W, X, Y$ and $Z$ are coefficients quadratic in the gauge field. Perturbation theory does not fix the diagonal terms of $A, W, C$ and $Y$. Normalization fixes the real part but the imaginary part remains ambiguous. Any choice to fix this ambiguity has to be local functions of the gauge fields. Computation of $S_{i}\left(A_{\mu}\right)$ in 2-D with $\mathrm{U}(1)$ gauge fields, $A_{\mu}$, gives

$$
\begin{aligned}
& \operatorname{Im}\left[S_{i}(A)-S_{i}(0)\right]=I_{1}+I_{2}+I_{3} \\
& I_{1}=\frac{1}{2} \operatorname{Tr}\left(A-A^{\dagger}+C^{\dagger}-C\right) \\
& I_{2}=\frac{1}{2} \operatorname{Tr}\left(W-W^{\dagger}+Y^{\dagger}-Y\right) \\
& I_{3}=\frac{1}{16 \pi} \sum_{p}\left[\frac{p_{1}+i p_{2}}{p_{1}-i p_{2}} z_{p}^{*} z_{-p}^{*}-\frac{p_{1}-i p_{2}}{p_{1}+i p_{2}} z_{p} z_{-p}\right] \\
& z_{p}=\int d^{2} x e^{i p x}\left[A_{1}(x)+i A_{2}(x)\right]
\end{aligned}
$$

$I_{1}$ and $I_{2}$ are completely ambiguous. $I_{3}$ is completely fixed and nonlocal. To make $\operatorname{Im}\left[S_{i}(A)-\right.$ $\left.S_{i}(0)\right]=0$, the diagonal terms of $A, W, C$ and $Y$ have to be chosen so that $I_{1}=0$ and $I_{2}$ cancels $I_{3}$. This cannot be achieved due to the nonlocality of $I_{3}$. The anomaly is a consequence of the non-local nature of $I_{3}$. Any choice for the diagonal terms in $W$ and $Y$, that is local, is a good choice and will not affect the anomaly. 
One choice is Brillouin-Wigner perturbation theory where $U<0 \pm \mid 0 \pm>_{1}$ is chosen to be real. The resulting formula for the effective action is

$e^{S_{i}(U)}=\frac{U<0-\mid 0+>_{U}}{1<0-\mid 0+>_{1}} e^{i\left[\Phi_{+}(U)-\Phi_{-}(U)\right]}$

$e^{i \Phi_{ \pm}(U)}=\frac{U<0 \pm \mid 0 \pm>_{1}}{|U<0 \pm| 0 \pm>_{1} \mid}$

This formula was shown to reproduce the correct continuum anomaly in [8] by computing (18) on several finite lattices for some fixed gauge fields and taking the continuum limit.

(18) has a very interesting property of becoming zero for some gauge fields. This can happen as follows. In a finite lattice $\mathbf{H}_{ \pm}$are both finite dimensional matrices. The ground state is obtained by filling all the negative energy states of $\mathbf{H}_{ \pm}$. The overlap of the two ground states will be non-zero only if the number of negative energy states of $\mathbf{H}_{ \pm}$are the same. In the gauge free case, half of the states are negative for both $\mathbf{H}_{ \pm}$. This situation is expected to be true for perturbative gauge fields. Here the overlap will be non-zero. One can come up with gauge field configurations where this is not the case [8]. Fluctuations around these gauge configurations cannot change this property. Since the overlap is zero due to a mismatch between the number of negative energy states of $\mathbf{H}_{ \pm}$, it is easy to see that insertion of an appropriate number of fermion operators between the two ground states will make it non-zero. Therefore, the effective action, (18), reproduces the 't Hooft picture [3] of the chiral determinant. It can be proven that $\mathbf{H}_{-}$always has an equal number of positive and negative eigenvalues for all gauge fields [8]. Only $\mathbf{H}_{+}$can have different number of positive and negative eigenvalues. Therefore (18) is well-defined for a generic gauge field configuration.

The success of this apporach to chiral fermions depends upon how well (18) reproduces all the physical results. The interesting point about (18) is that it interlocks two important features of chiral gauge theories: anomalies and fermion number violation. Since it is an overlap of two different states, it can have a phase and reproduce the anomaly [8]. Since it is an overlap of two different second quantized states, it can be exactly zero for some gauge configurations [8]. It is relatively easy to reproduce the anomaly in a conventional approach to chiral gauge theories but to reproduce fermion number violating processes on the lattice is hard because the formalism has to be valid for rough gauge fields [15.

Acknowledgements: I would like to thank Herbert Neuberger for a joyous and fruitful collaboration [6] 8]. I would also like to thank Sinya Aoki, Mike Creutz, Maarten Golterman, Jan Smit, Pierre van Baal, and Jeroen Vink for several interesting discussions.

\section{REFERENCES}

1. For a recent review of the various approaches see D. N. Petcher, Nucl. Phys. B(Proc. Suppl.) 30 (1993) 50.

2. For a classic review of fermions on the lattice see J. Smit, Acta Phys. Pol. B17 (1986) 531; Nucl. Phys. B(Proc. Suppl.) 4 (1988) 451.

3. G. 't Hooft, Phys. Rev. Lett. 37 (1976) 8; Phys. Rev. D14 (1976) 3432.

4. D. B. Kaplan, Phys. Lett. B288 (1992) 342.

5. S. A. Frolov and A. A. Slavnov, Phys. Lett. B309 (1993) 344.

6. R. Narayanan and H. Neuberger, Phys. Lett. B 302 (1993) 62;

7. R. Narayanan and H. Neuberger, RU-93-25 preprint (To appear in Nucl. Phys. B)

8. R. Narayanan and H. Neuberger, Phys. Rev. Lett. 71 (1993) 3251.

9. Y. Shamir, WIS-92/97; WIS-93/20; WIS93/56; WIS-93/57; WIS-93/99.

10. C. P. Korthals-Altes, S. Nicolis and J. Prades, CPT-93/P.2920

11. S. Aoki and H. Hirose, University of Tsukuba preprint, September 1993.

12. M. F. L. Golterman, K. Jansen, D. N. Petcher and J. C. Vink, UCSD/PTH 93-28, September 1993 .

13. C. G. Callan, Jr., and J. A. Harvey, Nucl. Phys. B250 (1985) 427.Calvey

14. D. B. Kaplan, Nucl. Phys. B(Proc. Suppl.) 30 (1993) 597.

15. W. Bock, J. Smit and J. C. Vink, Amsterdam ITFA preprints, 93-13; 93-18. 